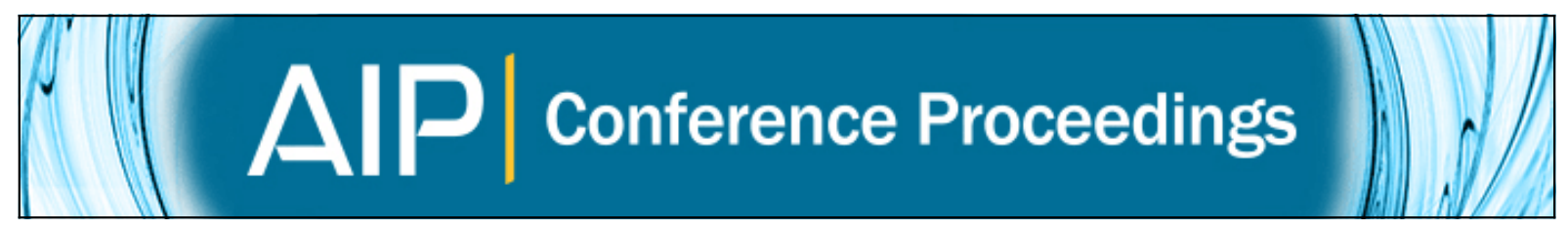

\title{
High resolution TVD schemes for interface tracking
}

K. Nandi, S. P. Walker, and A. W. Date

Citation: AIP Conference Proceedings 1738, 480072 (2016); doi: 10.1063/1.4952308

View online: http://dx.doi.org/10.1063/1.4952308

View Table of Contents: http://scitation.aip.org/content/aip/proceeding/aipcp/1738?ver=pdfcov

Published by the AIP Publishing

\section{Articles you may be interested in}

A silicon strip detector system for high resolution particle tracking in turbulence

Rev. Sci. Instrum. 72, 4348 (2001); 10.1063/1.1416112

High resolution tracking devices based on capillaries filled with liquid scintillator

AIP Conf. Proc. 450, 200 (1998); 10.1063/1.56970

A novel scheme of laser interferometer-refractometer with high spatial and temporal resolutions

Rev. Sci. Instrum. 68, 4353 (1997); 10.1063/1.1148396

On high-resolution finite volume shock capturing schemes

AIP Conf. Proc. 208, 940 (1990); 10.1063/1.39402

Recent developments in high-resolution tracking devices

AIP Conf. Proc. 163, 194 (1987); 10.1063/1.36928 


\title{
High Resolution TVD Schemes for Interface Tracking
}

\author{
K Nandi ${ }^{\mathrm{a}}$, S P Walker ${ }^{\mathrm{a}}$ and A W Date ${ }^{\mathrm{b}}$ \\ ${ }^{a}$ Dept. of Mechanical Engineering, Imperial College, South Kensington, London, UK, SW7 $2 A Z$ \\ ${ }^{b}$ Dept. of Mechanical Engineering, IIT Bombay, Powaii, Mumbai, India, 400076
}

\begin{abstract}
A first order upwind difference scheme (UDS) is routinely adopted for representing convection terms in a discretised space. UDS provides stable solutions. However it also introduces false diffusion in situations in which the flow direction is oblique relative to the numerical grid or when the cell-Peclet number is large. In order to predict sharp interface, higher order upwind schemes are preferred because of they reduce numerical dissipation. In interfacial flows, density and viscosity vary sharply in space. Representation of convective terms by Total variation diminishing (TVD) schemes ensures reduced smearing without impairing convergence property. TVD schemes develop formulae for interpolation of a cell-face value of the transported variable. If the interpolated value is bounded by the neighbouring nodal values then the scheme is 'Bounded'. However, not all TVD schemes possess this property of 'Boundedness'. The Normalised Variable Diagram (NVD) defines a domain within which the TVD scheme is bounded. Thus by combining the features of both TVD schemes and ensuring that they fall with the defined area of NVD, the convergence as well as the boundedness of a computational scheme can be ensured. In this paper, six different higher order schemes are considered some which are TVD bounded or unbounded, to solve the well known interface tracking problem of Rayleigh-Taylor Instability. To the best of our knowledge, a comparison of combined TVD/NVD principles in the case of interface tracking problems has not been reported in published literature.
\end{abstract}

Keywords: Interface tracking, TVD, SIMPLE, Collocated Grid

PACS: Replace this text with PACS numbers; choose from this list: http://www.aip.org/pacs/index.html

\section{INTRODUCTION}

The correct modelling of convection without excessive artificial dissipation while retaining high-order accuracy, stability and boundedness plays a key role in reproducing complex flow physics, such as sharp gradients or shocks in case of compressible flows. First order upwind difference produces stable and bounded solutions, but it introduces false diffusion especially in situations in which the flow direction is oblique relative to the numerical grid or when the cell-peclet number is large. The latter situation is found in prediction of interfacial flows of two immiscible fluids and the predicted interface is smeared. So in order to predict sharp interface, higher order upwind schemes are preferred because of they reduce numerical dissipation; however it also generates oscillations when it encounters steep variations of transported properties. Different researchers have proposed various techniques for capturing a well-defined interface, excellent reviews can be found in Rudman (1997), Tryggvassion (2001). One approach to the problem of preserving interface resolution is to discretise the convective scalar transport equation for the volume fraction with a differencing scheme that guarantees physical (bounded) volume fraction values while preventing the smearing of the transitional area over several mesh intervals [Hirt \& Nicolls(1981)]. It takes the interface orientation into account when calculating the amount of fluid fluxed over the face of a control volume, ensures physical volume fraction values (overall boundedness between zero and one), and keeps the transitional area over one control volume. However, it does not preserve local boundedness; i.e., a volume fraction value which initially lies between the values of its neighbours does not necessarily preserve this property when advected. High resolution differencing schemes such as TVD methods [Andrillon \& Allesandrini (2004)], Flux Corrected Transport (FCT) [Zalesak (1979)] schemes, and techniques using the Normalised Variable diagram (NVD) [Leonard (1991)] offer an alternative. Total variation diminishing (TVD) schemes possess several attractive features, such as delivering well-resolved, non-oscillatory discontinuities and boundedness. Boundedness is very important for convective discretised scheme in numerical computation, with which the numerical scheme will never surpass the maximum or minimum values inherently determined by the physical process itself [Leonard (1991)]. The NVA does not guarantee convergence of the proposed differencing schemes but tackles the problem of boundedness in a more general way. In this paper a novel interface capturing scheme [Nandi \& Date (2009)] is used to predict the interface and different higher order schemes are compared making use of the NVD concept. Thus by combining the features of both TVD schemes and 
ensuring that they fall with the defined area of NVD, the convergence as well as the boundedness of a computational scheme can be ensured.

\section{Numerical Formulation}

\section{Governing Equations}

$$
\begin{gathered}
\frac{\partial u_{j}}{\partial x_{j}}=\nabla \cdot \vec{V}=0 \\
\frac{\partial \rho_{m} u_{i}}{\partial t}+\frac{\partial \rho_{m} u_{j} u_{i}}{\partial x_{j}}=\frac{\partial}{\partial x_{j}}\left[\mu_{m} \frac{\partial u_{i}}{\partial x_{j}}\right]-\frac{\partial p}{\partial x_{i}}+\rho_{m} g_{i}+F_{s t, i} \delta+\frac{\partial}{\partial x_{j}}\left[\mu_{m} \frac{\partial u_{j}}{\partial x_{i}}\right]
\end{gathered}
$$

Equation 1 represents volume conservation whereas equation 2 represents momentum equation in conservative form. $F_{s t, i} \delta$ is the surface tension force that acts at the interface and $g_{i}$ is the gravitational acceleration and $\rho_{m}$ is a superficial density associated with the interface which represents a plane of discontinuity between the fluids. $\rho_{m}$ equals $\rho_{a}$ or $\rho_{b}$ within each fluid. Equations 1 and 2 can be derived from conventional control-volume analysis. They define fluid motion completely when procedures for evaluation of superficial properties $\rho_{m}$ and $\mu_{m}$ and the surface tension force $F_{s t, i} \delta$ is properly formulated. The interface location is tracked by solving an equation for the conserved scalar $\Phi$,

$$
\frac{\partial \Phi}{\partial t}+\frac{\partial \Phi u_{j}}{\partial x_{j}}=0
$$

The unsteady flows are calculated by solving the momentum, pressure correction and any scalar equations in a fully implicit manner on a staggered grid adopting SIMPLE algorithm by Patankar and Spalding (1971). Here, however the SIMPLE algorithm is implemented on collocated grids using a pressure smoothing term while retaining the fully implicit treatment.

\section{NVD Diagram}

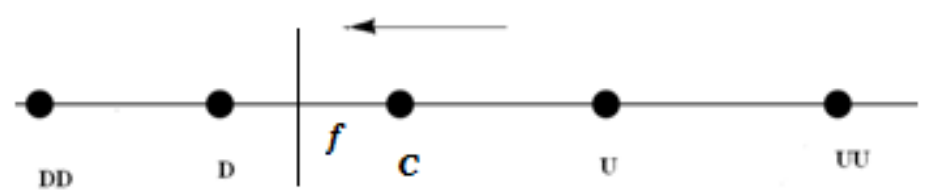

FIGURE 1. Typical node $P$ with upwind and downwind nodes.

Consider the variation of a convected scalar $\varphi$ in a $1 \mathrm{D}$ case, the normalized variable is defined as $\tilde{\phi}=\frac{\phi-\phi_{U}}{\phi_{D}-\phi_{U}}$, with this definition, any differencing scheme using only the nodal values of $\varphi$ at points $\mathrm{U}, \mathrm{C}$ and $\mathrm{D}$ to evaluate the face value can be written in the form: $\tilde{\phi}_{C}=\frac{\phi_{C}-\phi_{U}}{\phi_{D}-\phi_{U}}$ and $\tilde{\phi}_{f}=\frac{\phi_{f}-\phi_{U}}{\phi_{D}-\phi_{U}}$.

In order to avoid unphysical oscillations in the solution, we will require $\varphi_{C}$ (and consequently $\varphi_{f}$ ) to be locally bounded between $\varphi_{U}$ and $\varphi_{D}$, meaning either $\varphi_{U} \leq \varphi_{\mathrm{C}} \leq \varphi_{D}$ or $\varphi_{U} \geq \varphi_{\mathrm{C}} \geq \varphi_{D}$. If this criterion is satisfied for every point in the domain, the entire solution will be free of any unphysical oscillations. This is the basis of NVA. Gaskel and Lau (1988) showed that the boundedness criterion can be represented in a diagram by plotting $\varphi_{f}$ as a function of $\varphi_{c}$. This is known as the Normalised Variable Diagram or NVD. Thus by combining the features of both TVD schemes and ensuring that they fall with the defined area of NVD, the convergence as well as the boundedness of the scheme used for computation is ensured. In table i) $\phi_{f}$ as $f\left(\phi_{C}\right)$ of the six schemes compared are stated. 


\section{Results}

The different higher order convection schemes discussed in the previous section were applied to the problem of Rayleigh-Taylor instability. This cases was chosen as the representative cases since there is considerable stretching and distortion of the interface and is a well-known problem and computed by many researchers. Figure 2 shows the comparison of $\mathrm{F}$ distributions $(0.025<\mathrm{F}<0.0975)$ obtained by TVD [Lin Lin (1997)] and UDS schemes on 80x240 grid. The figure confirms that TVD predictions show much less smearing than the UDS scheme. The evolution of the interface, computed using six different higher order convection schemes is shown in Figure 3. The number of iterations per time step were kept same for comparison purposes. The volume error at the end of the schemes, were more or less the same $(\approx 0.4 \%)$.

TABLE 1. $\phi_{f}$ vs $\phi_{C}$ For Various Higher Order Schemes

\begin{tabular}{|c|c|c|}
\hline $\begin{array}{c}\text { High } \\
\text { Resolution } \\
\text { Schemes }\end{array}$ & Expression & Remarks \\
\hline $\begin{array}{l}\text { LIN LIN } \\
\text { [Lin (1997) ] }\end{array}$ & $\begin{aligned} \phi_{f} & =\phi_{c} \quad \text { if } \quad \phi_{c} \in[0,3 / 10] \\
& =3 / 8-\phi_{c} / 4 \quad \text { if } \phi_{c} \in[3 / 10,5 / 6] \\
& =1-\phi_{c} \quad \text { if } \phi_{c} \in[5 / 6,1] \\
& =0 \quad \text { if } \phi_{c} \notin[0,1]\end{aligned}$ & Bounded \\
\hline $\begin{array}{l}\text { HLPA } \\
\text { [Zhu (1991) ] }\end{array}$ & $\begin{aligned} \phi_{f} & =0 \quad \text { if } \quad \phi_{c} \notin[0,1] \\
& =\phi_{c}\left(1-\phi_{c}\right) \quad \text { if } \quad \phi_{c} \in[0,1]\end{aligned}$ & Bounded \\
\hline $\begin{array}{l}\text { MINMOD } \\
\text { [Zhu \& Rodi } \\
(1991)]\end{array}$ & $\begin{aligned} \phi_{f} & =0.5 \phi_{c} \quad \text { if } \quad \phi_{c} \in[0,0.5] \\
& =0.5-0.5 \phi_{c} \quad \text { if } \quad \phi_{c} \in[0.5,1] \\
& =0 \quad \text { elsewhere }\end{aligned}$ & Bounded \\
\hline $\begin{array}{l}\text { OSHER } \\
\text { [Chakravarthy } \\
(1983)]\end{array}$ & $\begin{aligned} \phi_{f} & =0.5 \phi_{c} \quad \text { if } \quad \phi_{c} \in[0,2 / 3] \\
& =1-\phi_{c} \quad \text { if } \quad \phi_{c} \in[2 / 3,1] \\
& =0 \quad \text { elsewhere }\end{aligned}$ & Bounded \\
\hline $\begin{array}{l}\text { SMART } \\
{[\text { Gaskell }} \\
(1988)]\end{array}$ & $\begin{aligned} \phi_{f} & =2 \phi_{c} \quad \text { if } \quad \phi_{c} \in[0,1 / 6] \\
& =0.375-0.25 \phi_{c} \quad \text { if } \quad \phi_{c} \in[1 / 6,5 / 6] \\
& =1-\phi_{c} \quad \text { if } \phi_{c} \in[5 / 6,1] \\
& =0 \quad \text { eslewhere }\end{aligned}$ & Unbounded \\
\hline $\begin{array}{l}\text { HOAB } \\
{[\text { Wei (2003) ] }}\end{array}$ & $\begin{aligned} \phi_{f} & =2.5 \phi_{c} \quad \text { if } \quad \phi_{c} \in[0,1 / 6] \\
& =0.5-0.5 \phi_{c} \quad \text { if } \quad \phi_{c} \in[1 / 6, .5] \\
& =0.25 \quad \text { if } \phi_{c} \in[0.5,0.75] \\
& =1-\phi_{c} \quad \text { if } \phi_{c} \in[0.75,1] \\
& =\quad \text { elsewhere }\end{aligned}$ & Unbounded \\
\hline
\end{tabular}



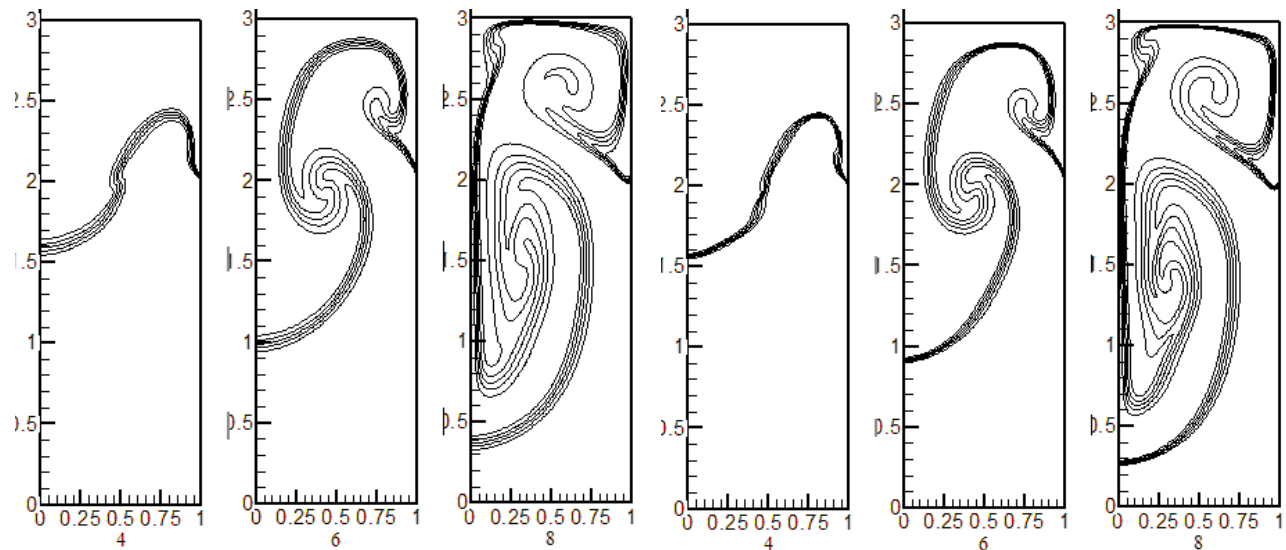

FIGURE 2. Comparison of UDS (left) and Lin-Lin TVD (right) predictions on $80 \mathrm{x} 240$ grid. F distributions $(0.025<\mathrm{F}<0.0975$, 10 contours).

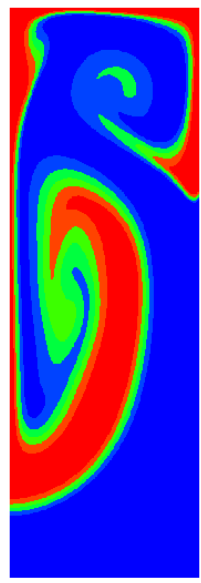

HLPA

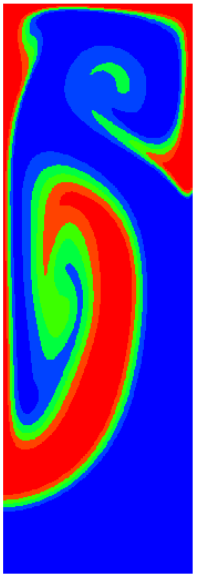

MINMOD

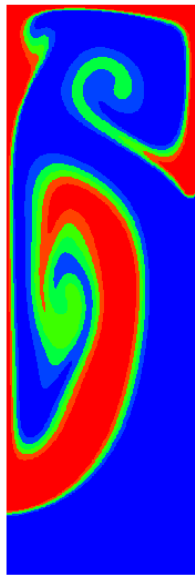

SMART

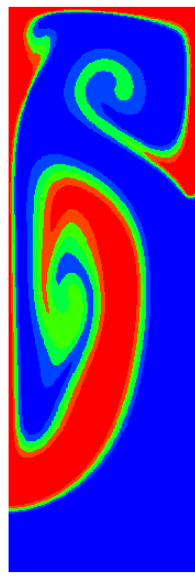

OSHER

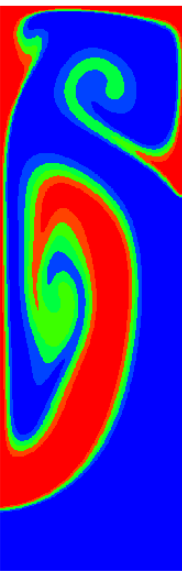

LINLIN

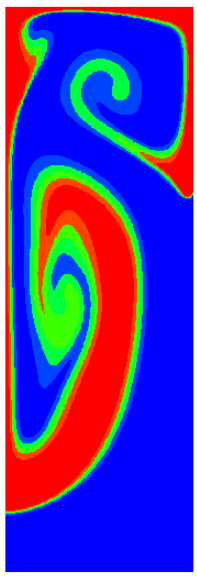

HOAB

FIGURE 3. Results of Rayleigh Taylor Instability at $\tau=8$ using different higher order schemes.

\section{REFERENCES}

1. Andrillon Y. \& Alessandrini B. A2D+TVOF fully coupled formulation for the calculation of breaking free-surface flow. $J$ of Marine Sc. \& Tech., 2004 ; 8:159-168.

2. Chakravarthy S. R. Osher S. High resolution of the OSHER upwind scheme for the Euler equations. AIAA Paper 1983; 83: 83-94.

3. Hirt C. W. \& Nicolls B. D. Volume of fluid (VOF) method for the dynamics of free boundaries. J. Computational Physics, 1981; 39: 201-225.

4, Leonard B. P. Bounded high-resolution up wind multidimensional finite-volume convection-diffusion algorithms. Comp. Meth.in App. Mech.\& Engg., 1991; 88: 17-28.

5. Lin C. H. \& Lin C. A simple high-order bounded convection scheme to model discontinuities. AIAA Journal, 1997; 35: 563565 .

6. Nandi K. \& Date A. W. Formulation of fully implicit method for simulation of flows with interfaces using primitive variables, Int. J. Heat and Mass Transfer, 2009; 52: 3217-24.

7. Patankar S. V. and Spalding D. B. A calculation procedure for heat mass and momentum transfer in three-dimensional parabolic flows. Int.J. Heat and Mass Transfer, 1971; 15: 1787-1806

8. Rudman M. Volume-tracking methods for interfacial flow calculations. Int. J. Num. Meth. in Fluids 1997; 24:671-691.

9. Tryggvasson G., Bunner B., Esmaeeli A., Juric D. \& Al-Rawahi N. A front tracking method for the computations of multiphase flows. J. Computational Physics 2001;169: 708-759.

10. Wei J. J., Yu B., Tao W. Q., Kawaguchi Y. and Wang H. S. A new high order accurate and bounded scheme for incompressible flow. Numerical Heat Transfer B 2003; 43:19-41.

11. Zalesak S. T. Fully Multidimensional Flux Corrected Transport Algorithm for Fluids. J. Computational Physics 1979; 31: 335-362.

12. Zhu J. and Rodi W. A low-dispersion and bounded convection scheme. Comp. Meth, in App. Mech. \& Engg. 1991; 92: 87-96.

13. Zhu J. A. Low diffusive and oscillation-free convections scheme. Comm. in App. Num. Meth., 1991; 7: 225-232. 